\title{
Svetlana Efimova \\ Prosa im Plural? Versuch einer Mereologie des Prosabegriffes
}

\begin{abstract}
Prosen, - dachte Professor Z., indem er vom Singular in den Plural wechselte, - das ist eine zukünftige Äquivalenz all dieser Ficciones und Non-fictions, lasst uns nur der Prosa erlauben, zum zählbaren Substantiv zu werden. Zum Glück hat bereits die „Vierte Prosa“ den Anfang gemacht. Alexandr Žolkovskij, Für S. (Posvjaščaetsja S., 1991) ${ }^{1}$
\end{abstract}

\section{Prosa und Mereologie}

„Vierte Prosa“ (Četvërtaja prosa) - ohne diese sprachliche Provokation ist eine Geschichte des Prosabegriffes in der russischen Literatur kaum denkbar. Auf diese Weise betitelte der Dichter Osip Mandel’štam einen in den Jahren 1929/30 entstandenen Prosatext, wobei sein Titel im Original genauso verfremdend wie in der Übersetzung klingt. Neben der Implikation, dass es mehrere Prosen geben könnte, stoßen in dieser Formulierung mehrere Gebrauchsweisen des Prosabegriffes aufeinander: Ist damit ein „vierter“ Prosatext gemeint oder wird hier eine neue (,vierte“) Prosapoetik suggeriert? Denn ,Prosa“ ist nicht nur eine ästhetische und rhetorische Kategorie wie ,ungebundene Rede‘, ${ }^{2}$ sondern auch eine Bezeichnung für den jeweiligen Prosatext. Zugleich ist ,Prosa“ ein Sammelbegriff, der eine Vielfalt von Textgruppen unterschiedlichen Maßstabs konturieren kann, von der Gesamtheit aller existierenden Prosatexte bis zu Werkgruppen eines Autors oder einer Autorin. Mandel’štams „vierte Prosa“ aktiviert auch diese Spannung zwischen ,Prosa' als Einzeltext und Textmenge: Hat man es hier mit einer akzentuierten rhetorischen Figur totum pro parte zu tun oder ist durch die Benennung eines Teils gerade eine zusammengesetzte Größe als eine Reihe von ,vier Prosen' impliziert? Aus diesen Fragen an Mandel'štams Titel wird ersichtlich, wie der Begriff ,Prosa‘ eine Problematik von Zusammensetzung und Teilung, Teil und Gesamtheit, Singular und Plural mit sich bringt. Von dieser Beobachtung ausgehend werde ich im Folgenden herausarbeiten, welches Einteilungspotenzial die

\footnotetext{
1 Alexandr Žolkovskij: Posvjaščaetsja S., in: ders.: NRZB. Rasskazy, Moskau 1991, 4-12, hier: 7. Meine Übersetzung - S.Е. „Прозы, - переходя с единственного числа на множественное, подумал профессор 3., - вот будущий эквивалент всех этих ficciones и non-fictions, дайте только прозе сделаться исчисляемым существительным, благо начало уже положено ,Четвертой прозой““. 2 Diese tradierte Minimaldefinition von Prosa bezeichnet als Ausgangspunkt ,das Fehlen von Metrum und metrischem Rhythmus“ in Abgrenzung zur Versdichtung. Vgl. Klaus Weissenberger: Art. „Prosa“, in: Historisches Wörterbuch der Rhetorik, hrsg. von Gert Ueding, Tübingen 1992-2015, Bd. 7, 321-348, hier: 321.
}

๖ Open Access. ๑ 2021 Svetlana Efimova, publiziert von De Gruyter.

(G) BY-ND Dieses Werk ist lizenziert unter der Creative Commons Attribution-NoDerivatives 4.0 International Lizenz.

https://doi.org/10.1515/9783110729085-006 
Prosakategorie in sich birgt und welche (An-)Ordnungsmodelle sie im Bereich der literarischen Textualität stiftet.

In den Vordergrund gerät dabei eine mereologische Dimension des Prosabegriffes: Mereologie (von griech. meros ,Teil') ist ein Gebiet von Philosophie, Logik und Mathematik, das diverse Teil-Ganzes-Relationen sowie Beziehungen zwischen den Teilen eines Ganzen ergründet. Im Rahmen der Philosophie setzt sich Mereologie mit ontologischen und erkenntnistheoretischen Aspekten der Wirklichkeitsstrukturierung auseinander. Obwohl sich mereologisches Denken seit Platon und Aristoteles beobachten lässt, gilt Edmund Husserls dritte Untersuchung Zur Lehre von den Ganzen und Teilen aus dem zweiten Band der Logischen Untersuchungen (1901/1913) als die erste systematische Theorie dieser Art. ${ }^{3}$ In seiner Einleitung zur dritten Untersuchung führt Husserl eine Reihe der „formalen gegenständlichen Kategorien“ an, die zum breiten Bereich der Teil-Ganzes-Problematik gehören: „Ganzes und Teil, Subjekt und Beschaffenheit, Individuum und Spezies, Gattung und Art, Relation und Kollektion, Einheit, Anzahl, Reihe, Ordinalzahl, Größe usw. “4 In diesem Themenfeld lässt sich auch Prosa als Textart, textuelle Beschaffenheit und Textreihung bis zum Einsatz einer Ordinalzahl (,vierte“) verorten. ${ }^{5}$

Im Folgenden wird mit Rückgriff auf Husserls mereologische Lehre untersucht, wie der Prosabegriff Relationen zwischen einem Teil und einem Ganzen sowie zwischen den Teilen eines Ganzen auf drei Ebenen konfiguriert: zwischen Literatur und anderen Diskursen, zwischen mehreren literarischen Texten und innerhalb eines Prosatextes. Dabei werde ich induktiv vorgehen und eine Reihe von paratextuellen und metaliterarischen Prosareflexionen aus dem 20. Jahrhundert analysieren, die als Selbstzuschreibungen an der Schnittstelle zwischen Theorie und Schreibpraxis liegen.

3 Vgl. Lothar Ridder: Mereologie. Ein Beitrag zur Ontologie und Erkenntnistheorie, Frankfurt a. M. 2002, 1. Der Begriff ,Mereologie‘ wurde in den 1920er Jahren vom polnischen Mathematiker und Philosophen Stanisław Leśniewski geprägt.

4 Edmund Husserl: Logische Untersuchungen, Bd. 2. Teil 1. Untersuchungen zur Phänomenologie und Theorie der Erkenntnis, 2., umgearb. Aufl., Halle (Saale) 1913, Nachdruck als 7. Aufl., Tübingen 1993, 225. Diese Kategorien gehören zu einer „,reinen (apriorischen) Theorie der Gegenstände als solcher“ (ebd.). Husserl betont, dass er die Begriffe ,Gegenstand“ und ,Teil“ „im weitesten Sinne“ verwendet (S. 228). Darunter können auch Sachverhalte und Eigenschaften subsumiert werden.

5 Dass die mereologische Problematik ein Kerngebiet der literaturwissenschaftlichen Studien trifft, wurde bereits 1990 von Lubomír Doležel hervorgehoben: Für ihn ist die „Vorstellung von Literatur als Struktur“ bereits seit Aristoteles mit einem „mereologische[n] Modell der Poetik“ verbunden. Lubomír Doležel: Geschichte der strukturalen Poetik. Von Aristoteles bis zur Prager Schule [1990], übers. von Norbert Greiner, Dresden u. München 1999, 9. 


\section{Prosa zwischen Diskursen: Integration und Pluralisierung}

Die an Osip Mandel'štams Titel Vierte Prosa gestellten Fragen berühren ein verwobenes Assoziationsnetz und müssen als solche unbeantwortet bleiben. Der Dichter hat sich in den 1920er Jahren dem Prosaschreiben zugewandt; zu seinen Lebzeiten wurden in Buchform Das Rauschen der Zeit (Šum vremeni, 1925), Die ägyptische Briefmarke (Egipetskaja marka, 1928) und die Essaysammlung Über Dichtung (O poėzii, 1928) publiziert. Drei existierende Deutungsansätze zum rätselhaften Titel gehen auf die Memoiren von Nadežda Mandel'štam zurück: Vierte Prosa, die posthum veröffentlicht wurde, sei das vierte Prosawerk Mandel'štams und erinnere zudem an den vierten Stand (das Proletariat) und an das ,Vierte Rom ${ }^{6}{ }^{6}$

Dass das Echo der Vierten Prosa bis heute in der russischen Literatur widerhallt, wird deutlich, wenn Pluralisierungen von ,Prosa‘ Mandel’štams Titel als Prätext aktualisieren. ${ }^{7}$ Die Verwandlung von ,Prosa ${ }^{‘}$ in ein zählbares Substantiv wurde auch vom Literaturwissenschaftler und Schriftsteller Alexandr Žolkovskij aufgegriffen: Als Einstieg zu meinem Aufsatz dient ein Zitat aus seiner Geschichte Für S. (Posvjaščaetsja S.), die zur kleinen Erzählsammlung Unleserlich (NRZB, 1991) gehört. ${ }^{8}$ Die Handlung spielt Ende der 1980er Jahre in den USA; die autofiktionale Hauptfigur ist ein russischer Literaturprofessor, der an einer Universität in Kalifornien unterrichtet und dessen Name sich hinter dem Buchstaben Z. verbirgt. Nachdem dieser Professor Z. seinen berühmten Landsmann Iosif Brodskij bei einem Literaturabend in New York gesehen hat, fällt ihm ein, dass der Dichter Brodskij neulich sein erstes Prosabuch veröffentlichte, die englischsprachige Essaysammlung Less Than One. Danach versucht Z. vergeblich, diesen Band in drei großen Buchhandlungen in Manhattan zu finden; er wird von einer Abteilung in die andere geschickt: „Neuerscheinungen, LiteraturKlassiker, Belletristik, Biographien, Dokumentarprosa“" - die Prosa von Brodskij ist

\footnotetext{
6 Vgl. Nadežda Mandel’štam: Vospominanija, Moskau 1989, 166. Die Idee von Moskau als ,Drittes Rom' geht auf Schriften des Mönches Filofei aus dem 16. Jahrhundert zurück und ist mit der Annahme verbunden, dass es ein Viertes nicht geben wird: Somit hat die Ordinalzahl „vierte“ einen apokalyptischen Beiklang. In Mandel'štams Gedicht Der 1. Januar 1924 (1 janvarja 1924) ist ein Eid erwähnt, den das lyrische Ich dem „vierten Stand“ geschworen hat. Die soziale Bewegung der Klassen in der Revolutionszeit war eines der zentralen Argumente für das „Ende des Romans“ in Mandel'štams gleichnamigen Essay (Konec romana) von 1922: Eine Krise der Persönlichkeit in der Geschichte führt einen Prosaiker vom Roman zur Chronik.

7 Hingewiesen sei etwa auf die neueren Sammelausgaben Zweite Prosa von Viktor Sosnora (Vtoraja Prosa, 2018) und Drei Prosen von Michail Šiškin (Tri prozy, 2012).

8 „NRZB“ ist eine russische Abkürzung, die bei Editionen der handschriftlichen Manuskripte für ,unleserlich' steht.
}

9 Žolkovskij, Posvjaščaetsja S. (Anm. 1), 6. 
nicht da. Gekauft wird im Endeffekt ein anderes Buch, auf das Z. beim Stöbern unter dem Buchstaben B gestoßen ist: Borges' Ficciones. Als er mit diesem Buch im Flugzeug nach Los Angeles sitzt und darüber nachdenkt, dass es ein Fehler war, Brodskijs Essays als Non-Fiction neben den Erzählungen von Borges zu suchen, fällt ihm der zitierte Gedanke ein: „Prosen [...] - das ist eine zukünftige Äquivalenz all dieser Ficciones und Non-fictions, lasst uns nur der Prosa erlauben, zum zählbaren Substantiv zu werden." ${ }^{10}$ Diese ,Prosa“ ist zwar ein Klassifikationsbegriff, fordert aber zugleich tradierte Ordnungskategorien heraus, die in diesem Fall durch die Abteilungen der Buchhandlung verkörpert werden. Die Sammelbezeichnung ,Prosen` konturiert eine Menge, erzeugt ein textuelles Feld jenseits der fragwürdig gewordenen Abgrenzung von Gattungen, Wissensbereichen, von Fiktion und Fakt bzw. Sachlichkeit.

Prosa als ein alternatives Klassifikationsinstrument legt die Frage nahe, ob diese Zuschreibung an den Grenzen des Literarischen aufhört oder sich weiterhin in die anderen Diskurse erstreckt. Für Žolkovskij schaffte der Prosabegriff vor allem einen interdiskursiven Übergang zwischen dem literaturwissenschaftlichen und literarischen Schreiben: Als bekannter Slavist und Strukturalist publizierte er seit den 1990er Jahren zunehmend Sammlungen essayistischer Kurzprosa und inszenierte sich erfolgreich als Prosaiker. Auch Mandel'štams Vierte Prosa, die sich kaum einer Gattung zuordnen lässt, transzendiert interdiskursive Grenzen zwischen Literatur und Publizistik: Sie enthält Elemente eines offenen Briefes, eines Pamphlets und einer Schmährede gegen die politisch konforme Literatur. Vierte Prosa aktualisiert auch eine mediale Dimension der Prosakategorie, die Grenzen zwischen Mündlichkeit und Schriftlichkeit überschreitet, indem der autobiographische Ich-Erzähler behauptet: „Ich habe keine Manuskripte, keine Notizbücher, keine Archive. Ich habe keine Handschrift, weil ich niemals schreibe."11

Die umfangreichste Konstellation des Prosabegriffes ist also ein potenziell allumfassender Textbereich jenseits der Grenzen zwischen Mündlichkeit und Schriftlichkeit, Sachtext und Kunst. Die Breite des abgedeckten Textfeldes steht in einem reziproken Verhältnis mit der Vagheit des jeweiligen Prosakonzepts: Je größer die Gesamtmengen konturiert werden, desto schwieriger wird der Schritt von einer summativen zu einer integrativen Prosakategorie. Daraus ergibt sich auch die Frage, wie sich die literaturwissenschaftliche Optik verändern würde, wenn Texte primär als Prosen betrachtet würden. Und zu erwägen ist, ob die heuristische Pluralform ,Prosen`

10 Ebd., 7.

11 Ossip Mandelstam: Das Rauschen der Zeit. Gesammelte „autobiographische“ Prosa der 20er Jahre, übers. von Ralph Dutli, Zürich 1991, 258. „У меня нет рукописей, нет записных книжек, архивов. У меня нет почерка, потому что я никогда не пишу.“ Osip Mandel’štam: Sobranie sočinenij v četyrëch tomach, Bd. 3, Moskau 1994, 171. Zu einem autobiographischen, literarischen und medienanthropologischen Hintergrund dieser polemischen Geste vgl. Svetlana Efimova: Das SchriftstellerNotizbuch als Denkmedium in der russischen und deutschen Literatur, Paderborn 2018, 22, 267 f. Seit der antiken Rhetorik umfasst der Prosabegriff auch die mündliche Rede. 
auch eine Möglichkeit bieten würde, über Pluralität und Zusammenspiel von unterschiedlichen Schreibweisen nachzudenken.

Žolkovskijs Sammlung Unleserlich endet mit dem Essay Zwischen Gattungen (Meždu žanrami), der einem Auszug aus Lidija Ginzburgs Buch Literatur auf der Suche nach der Realität (Literatura v poiskach real'nosti, 1987) gewidmet ist und somit auf eine Tradition für Žolkovskijs Prosa(konzept) hindeutet. Lidija Ginzburg (1902-1990), eine Schülerin der russischen Formalisten, wurde in der Sowjetunion als Literaturforscherin bekannt und verfasste seit den 1920er Jahren Erinnerungsprosa, deren Publikation erst in den 1980er Jahren möglich geworden ist. Ihr Band Literatur auf der Suche nach der Realität ist eine Sammlung, die in zwei Teile gegliedert ist: Der erste Teil umfasst fünf wissenschaftliche Aufsätze, die sich insgesamt um die Problematik des Realismus und der Historizität von Literatur drehen. Der zweite Teil besteht aus sieben weiteren Texten; er beginnt mit Memoiren, mit Prosa autobiographischen Charakters, dann geht er in die sogenannten „Aufzeichnungen“ („zapisi“) über und mündet in das bekannteste und vielfach übersetzte Prosawerk von Ginzburg: Die Aufzeichnungen des Blockademenschen (Zapiski blokadnogo čeloveka), eine literarische Verarbeitung des Kriegsalltags während der Blockade von Leningrad im zweiten Weltkrieg, die Ginzburg selbst überlebt hat. ${ }^{12}$ Die Verbindung zwischen den beiden Buchteilen wird im kurzen Vorwort, das dem Band vorausgeschickt wird, mithilfe des Prosabegriffes begründet:

Der erste Teil des Buches besteht aus den Aufsätzen theoretischen Charakters. [...] Der zweite Teil ist memoirenhaft und essayistisch. Solche Art von Literatur existierte seit je in meiner schriftstellerischen Praxis. Zwischen den Gattungen der beiden Teile verspüre ich keinen unüberwindbaren Bruch. Das sind für mich alles Spielarten der Prosa. ${ }^{13}$

„Spielarten [raznovidnosti] der Prosa“ - diese Formulierung impliziert dieselbe Verbindung von Integration und Pluralisierung, die im grammatischen NumerusPaar ,Prosa/Prosen“ miteingeschlossen ist. Dieser Hinweis von Ginzburg regt dazu an, die Texte ihrer Sammlung hinsichtlich eines konzeptuellen Zusammenhangs im Zeichen der Prosa zu lesen - und eben nicht bloß als mechanisch-summative Sammeledition.

Während Ginzburgs Aufsätze eine Realitätserfassung durch die Literatur als Thema haben, praktiziert sie im zweiten Teil einen prosaischen Erinnerungs- und

12 Am Ende der Aufzeichnungen des Blockademenschen stehen drei Daten als Arbeitsanläufe und als Signal für die Verflechtung zwischen Augenzeugenschaft und Gedächtnisarbeit: 1942 - 1962 - 1983.

13 Meine Übersetzung - S. Е. „Первая часть книги состоит из статей теоретического характера. [...] Вторая часть - мемуарная и эссеистическая. Подобный род литературы издавна существовал в моей писательской практике. Между жанрами обеих частей я не ощущаю непроходимого разрыва. Все это для меня разновидности прозы.“ Lidija Ginzburg: Literatura v poiskach real'nosti, Leningrad 1987, 2. 
Aufzeichnungsmodus. In diesem verarbeitet sie Ausschnitte aus einer historischen Zeitspanne, beginnend mit den 1920er bis hin zu den 1980er Jahren. Man kann nun gewisse stilistische Kontinuitäten im gesamten Buch verfolgen und das Zusammenspiel der wissenschaftlichen und literarischen Schreibgesten beobachten. Bei einem Versuch, diesen Hinweis auf „Spielarten der Prosa“ als Schreibweise zu explizieren, eröffnen sich mehrere Möglichkeiten: Ist ,Prosa‘ der analytisch-sachliche Stil, der auch Ginzburgs literarische Erinnerungen durchdringt, oder umgekehrt diejenige Wortkunst, die einige theoretische Passagen in die Nähe zum Literarischen rückt? Der Begriff ,Prosa“ dient hier weniger als eine fest definierte Zuschreibung, sondern vielmehr als ein heuristisches Medium der Selbstreflexion und als ein paratextuelles Instrument, um die Lektüre zu lenken: Damit werden unterschiedliche Texte und auch Diskurse als Spielarten in Bezug zueinander gesetzt und die Aufmerksamkeit auf die potenziellen Schwellenmomente der Hybridität gezogen. Der Begriff ,Prosa“ tritt hier in seiner Funktionalität hervor, potenzielle Schnittmengen im Bereich der tradierten Wissens- und Textordnungen zu konturieren; mehr als nur eine Legitimation von editorischen Sammlungsformaten, kann er eine orientierende Wirkung entfalten und dynamische Ordnungsstrukturen ermöglichen. Mereologisch gesehen bewirkt der Prosabegriff bei Ginzburg eine interdiskursive Gesamtkonstruktion und prägt ein Bewusstsein für das Verhältnis zwischen deren Teilen.

Die Betrachtung als Prosa lässt eine basale Textualität und eine damit verbundene Erkenntnisdimension des jeweiligen Textes (bei Ginzburg - „Suche nach der Realität“) in den Vordergrund treten, zugleich bleibt aber der Prosabegriff offen für Prosen im Sinne der pluralen Stile und Schreibweisen. Gerade in dieser Offenheit für Pluralität, die von einer integrativen Kraft untrennbar ist, liegt ein ästhetisches und epistemologisches Potenzial der Prosakategorie, das eine Mereologie der Prosa ermöglicht.

\section{Kompositionen: „prózy“ und Prosastücke}

Neben der Möglichkeit, interdiskursive Ordnungsstrukturen zu bewirken, besitzt der Prosabegriff eine weitere mereologische Dimension im engeren Rahmen des literarischen Diskurses, die sich durch einen kleinen linguistischen Exkurs einleiten lässt. Wir empfinden die grammatische Formbildung ,Prosen“ als sprachspielerisch, weil Prosa in den meisten europäischen Sprachen ein unzählbares Singularwort ist. Als Ausnahmen gibt es zumindest zwei eng verwandte slawische Sprachen, in denen Prosen als „prózy“ sprachlich kodifiziert sind, nämlich Tschechisch und Slowakisch. Ein Blick in die akademischen Wörterbücher der beiden Sprachen zeigt, dass dort gerade die Pluralform als Illustration der kodifizierten Bedeutung von próza als ,Prosawerk(e)` oft auftaucht. Als typisches Beispiel wird zumindest in drei unterschiedlichen Wörterbüchern dieselbe Wendung angeführt - „krátké prózy“ („kurze Prosen“); ein tschechisches Fremdwörterlexikon fügt eine Erklärung hinzu: „novely, povídky ap.“ 
(„Novellen, Geschichten u. Ä.“). ${ }^{14}$ Das ist einerseits nichts Überraschendes, wenn man an den bereits etablierten Begriff ,Kurzprosa' denkt, andererseits entsteht durch die Pluralform wiederum eine besondere Sensibilisierung für die Relation zwischen dem gesamten Textbereich und einem Einzeltext.

Eine wichtige Episode in der Geschichte der tschechischen Prosen ist mit der Prager Moderne verbunden: 1917 erschien in Prag Richard Weiners Sammlung Der gleichgültige Zuschauer und andere Prosen (Netečný divák a jiné prózy). Ein Jahr früher veröffentlichte derselbe Prager Verlag (Fr. Borový) ein anderes Buch mit dem ähnlichen Titelzusatz: Die leuchtenden Tiefen und andere Prosen (Zářivé hlubiny a jiné prózy, 1916). Diese Sammlung hatte eine Doppelautorschaft: Karel Čapek, damals ein junger Autor und später einer der bekanntesten tschechischen Prosaiker, und sein Bruder Josef Čapek, der auch als Maler und wichtiger Vertreter des Kubismus namhaft geworden ist. Drei Jahre davor publizierte Josef Čapek im Prager Almanach für das Jahr 1914 (Almanach na rok 1914) eine Textkomposition unter dem Titel Drei Prosen (Tři prózy), der uns unmittelbar an die Vierte Prosa erinnert, obwohl das vermutlich eine zufällige Koinzidenz ist. Diese Überschrift subsumiert drei kurze Texte Ein Spaziergang, Ein Ereignis und Eine Wasserlandschaft (Procházka, Událost und Vodní krajina): Wie die etwas schematisch klingenden Titel andeuten, treffen sich hier auf geringem Raum Abschnitte mit einem stärkeren und abgeschwächten narrativen Element als Variationen des Prosaschreibens.

Warum sind „Prosen“ in der tschechischen Moderne der 1910er Jahre aufgeblüht? In der germanistischen Kurzprosaforschung wird die überzeugende These vertreten, dass ,Prosa‘ als Bezeichnung bzw. Titel vor allem dort produktiv wird, wo sich ein Text einer Gattungszuschreibung entzieht. ${ }^{15}$ Die erwähnten tschechischen Beispiele würden diesen engeren Prosabegriff allerdings nicht immer bestätigen. So hat Jan Mukařovský in seinem Aufsatz Die Entwicklung von K. Čapeks Prosa argumentiert, dass der Band Die leuchtenden Tiefen im Grunde genommen aus Erzählungen (povídky) besteht und gerade Čapeks Übergang „,von der lyrischen zur erzählenden Prosa“ markiert. ${ }^{16}$ Der spätere Karel Čapek ist mit seinen Romanen, die Elemente von Antiutopie und ScienceFiction integrieren, eher kein Vertreter der hermetischen und antinarrativen Prosa. Sowohl die Brüder Čapek als auch der dem Expressionismus nahestehende Richard Weiner befanden sich zudem im Austausch mit dem zeitgenössischen Kubismus, der ein Bewusstsein für Formen, Flächen und Teil-Ganzes-Relationen schärfte: Ein

14 Vgl. Slovník slovenského jazyka, III. diel, p-r, Bratislava 1963, 645; Slovník spisovného jazyka českého, IV, P-Q, Praha 1989, 464; Nový akademický slovník cizích slov. A-Ž, Praha 2009, 663.

15 Vgl. Thomas Althaus, Wolfgang Bunzel, Dirk Göttsche: Ränder, Schwellen, Zwischenräume. Zum Standort Kleiner Prosa im Literatursystem der Moderne, in: dies. (Hrsg.): Kleine Prosa. Theorie und Geschichte eines Textfeldes im Literatursystem der Moderne, Tübingen 2007, IX-XXVII, hier insb.: XXIV.

16 Jan Mukařovský: Die Entwicklung von K. Čapeks Prosa [1934], in: ders.: Kapitel aus der Poetik, übers. v. Walter Schamschula, Frankfurt a. M. 1967, 55-107, hier: 59. 
Gegenstand konnte in geometrische Figuren zerlegt werden, woraus wiederum das Potenzial einer erneuten Synthetisierung entstand. Nach dem Urteil des Kritikers und Autors Miroslav Rutte wird in der Sammlung Die leuchtenden Tiefen und andere Prosen „literarischer Kubismus“ im Sinne einer „Auflösung der epischen Linie in verschiedene Schichten“ erkannt, die jedoch in ihrem Zusammenspiel wirksam werden. ${ }^{17}$ Diese Schichtung und Bindung entspricht dem editorischen Aufbau eines Prosabuchs aus einzelnen Prosen, die auf ursprünglich zerstreute Zeitungspublikationen zurückgehen. Anzumerken sei in dieser Hinsicht, dass Karel Čapeks erster Roman Die Fabrik des Absoluten (Továrna na absolutno, 1922) auch ein Fortsetzungsroman, ein „FeuilletonRoman“ (so der Untertitel) war und dass sein später Roman Der Krieg mit den Molchen (Válka s mloky, 1936) durch eine Collagetechnik und Integration heterogener Textarten und Wissensbereiche (wie Naturwissenschaft) gekennzeichnet ist.

Somit lässt sich annehmen, dass die Pluralform prózy in diesem Kontext für ein kombinatorisches Übergangsmoment und für die Erschaffung einer Komposition steht: Aus einzelnen Prosen wird Prosa. Wie auf der interdiskursiven Ebene sehen wir auch hier, wie der Prosabegriff Übergänge und Sammlungsstrukturen begünstigt, nicht nur im klassifikatorischen Sinne variabler Gesamtheiten, sondern jetzt primär im kompositorischen Sinne der textuellen Zusammensetzung als neue Größe. ${ }^{18}$

Wenn ,Prosa' auch für eine textuelle Anordnung unterschiedlicher Segmente von Montage bis editorischen Sammelformaten steht und die tschechische Pluralform prózy solche Segmente benennen kann, dann stellt sich die Frage, ob der Prosabegriff auch in einer anderen Sprache ähnlich funktionieren kann. Ein vergleichbarer Fall ist das Wort ,Prosastück', das vor allem durch Robert Walser metaliterarisch und paratextuell beinahe zu einem Gattungsbegriff etabliert wurde. 1916 erschien nicht nur das besprochene Prosen-Buch der Brüder Čapek in Prag: Dasselbe Jahr ist auf dem Umschlag der ersten Ausgabe von Robert Walsers Sammlung Prosastücke vermerkt, die 1917 in Zürich gedruckt wurde. Die Bezeichnung ,Prosastück' taucht auch als Selbstattribution immer wieder in Walsers Kurzprosatexten, die vereinzelt in Zeitungen und kompiliert in Sammlungen veröffentlicht wurden.

In der Walser-Forschung wird dieser Wortgebrauch von ,Stück' mereologisch gedeutet: Dadurch tritt eine Teil-Ganzes-Relation hervor, die sich einem serialen

17 Walter Schamschula: Geschichte der tschechischen Literatur, Bd. III, Von der Gründung der Republik bis zur Gegenwart, Köln 2004, 223.

18 Dies entspricht einer Feststellung von Thomas Althaus, Wolfgang Bunzel und Dirk Göttsche in Bezug auf das gesamte Textfeld der Kleinen Prosa: „Die meisten Genres Kleiner Prosa im 20. Jahrhundert sind jedenfalls auf nicht-kontingente Agglomeration, zum Teil sogar auf zyklische Komposition angelegt“. Althaus/Bunzel/Göttsche, Ränder (Anm. 15), XXI. Sie haben auch auf eine Annäherung des modernen Romans an eine „Ansammlung diverser Kleinformen“ und zugleich an einen „Schnittpunkt unterschiedlichster Diskurse“ hingewiesen: von einer Binneneinlage zur parzellierten Struktur und zur Collagetechnik (ebd.). Diese Tendenz ist für eine Mereologie der Prosa von großer Bedeutung. 
Schreiben verdankt. ${ }^{19}$ Wenn das ,Stück' einen Teil impliziert, dann lässt sich daraus eine Vorstellung von Prosa als einem Gefüge von Prosastücken ableiten. Diese Idee wird als Selbstinszenierung an einer berühmten Stelle in Walsers Prosastück Eine Art Erzählung (1928/29) verkündet:

Meine Prosastücke bilden meiner Meinung nach nichts anderes als Teile einer langen, handlungslosen, realistischen Geschichte. Für mich sind die Skizzen, die ich dann und wann hervorbringe, kleinere oder umfangreichere Romankapitel. Der Roman, woran ich weiter und weiter schreibe, bleibt immer derselbe und dürfte als ein mannigfach zerschnittenes oder zertrenntes Ich-Buch bezeichnet werden können. ${ }^{20}$

Eine „handlungslose Geschichte“ und ein unendlicher Roman, der immer „weiter und weiter“ fortgesetzt wird, könnten auch als ,Prosa“ im Singular bezeichnet werden. Ausschlaggebend ist, dass Unabgeschlossenheit gerade durch das Format eines kleinen Abschnitts ermöglicht wird: Teilung und Verbindung bilden ein dialektisches Doppelwesen von Prosa. Fortsetzung und Abbruch treffen in einer anderen paradigmatischen Reflexion Walsers aufeinander, Das letzte Prosastück (1919): „Wahrscheinlich ist dies mein letztes Prosastück““. ${ }^{21}$ Angekündigt wird ein Schlusspunkt nach zehn Jahren immer fortgesetzten Schreibens und zugleich erweist sich dieses vermeintliche Ende als der Anfang eines weiteren Abschnittes: „Was tat ich zehn Jahre lang? Um diese Frage beantworten zu können, muß ich [...] ein neues Kapitel oder frischen Abschnitt beginnen“.22 Dieses Spannungsverhältnis von Fortsetzung und Zäsur ist bereits in die inszenierte Komplementarität des Prosastücks und der Prosa („Ich-Buch“) eingeschrieben.

Was macht diese Summe oder Reihe von Stücken zu einer neuen Einheit? Bei Walser ist es wie im Falle von Lidija Ginzburg die Performativität des Paratextes, des Prosabegriffes selbst, der einen, homogenisierenden' Lektüremodus, eine Suche nach verbindenden Elementen (auch auf der textuellen Ebene) stiftet. Andererseits erinnert Walsers „handlungslose[], realistische[] Geschichte“ an den Begriff der ,sujetlosen Prosa“ (vnesjužetnaja/bessjužetnaja proza), der in den 1920er Jahren von Viktor Šklovskij herausgearbeitet wurde. Sein Aufsatz Zur Technik der sujetlosen Prosa erschien 1929 im Sammelband der Gruppe LEF („Linke Front der Künste“) Literatur des Faktums, der Programmatik einer nichtfiktionalen, faktographischen, d. h. dem Dokument und Journalismus nahestehenden Literatur festhielt. Dort skizzierte Šklovskij mehrere

19 Laut Michael Niehaus ist ein ,Prosastück“ „nicht für sich selbst stehend“, sondern beansprucht seinen Platz „in einer Umgebung“; er sei kein Werk, sondern ein Teil des Gesamtwerks. Michael Niehaus: Das Prosastück als Idee und das Prosastückverfassen als Seinsweise: Robert Walser, in: Althaus/Bünzel/Göttsche (Hrsg.), Kleine Prosa (Anm. 15), 173-186, hier: 174, 186.

20 Robert Walser: Eine Art Erzählung, in: ders.: Sämtliche Werke in Einzelausgaben, hrsg. von Jochen Greven, Bd. 20, Frankfurt a. M. 1986, 322-326, hier: 322.

21 Robert Walser: Das letzte Prosastück, in: ders.: Sämtliche Werke in Einzelausgaben, hrsg. von Jochen Greven, Bd. 16, Frankfurt a. M. 1985, 321-327, hier: 321.

22 Ebd. 
Optionen, eine Verbindung der Teile ohne narrative Sujetbildung zu leisten: eine zeitliche oder räumliche Bewegung (Memoiren oder Reiseliteratur), eine Verbindung der Textteile durch einen Erzähler statt durch eine Figur (Feuilleton). ${ }^{23}$ An diesem Zusammenschluss von Sujetlosigkeit und Faktographie wird ein subversives Potenzial der Prosakategorie ersichtlich: Ohne Narration und Fiktion als gewisse Dominanten des literarischen Diskurses komplett abzulehnen, fordert die Prosakategorie sie heraus, indem sie alternative Schreibarten stiftet. Die räumliche Bewegung und die suggerierte Identität desselben Erzählers als Elemente einer sujetlosen Prosa würden auch auf Walsers „Ich-Buch“ und die darin enthaltenen Prosastücke zutreffen, von denen viele mit dem Motiv des Spazierengehens verbunden sind.

\section{Prosa als Moment und Stück}

Das Wort ,Stück' ist Anfang des 20. Jahrhunderts bei Edmund Husserl zu einem mereologischen Begriff avanciert. In der bereits erwähnten dritten Untersuchung Zur Lehre von den Ganzen und Teilen aus den Logischen Untersuchungen unterscheidet er zwischen zwei apriorischen Typen von Teilen, Moment und Stück:

Wir fixieren zunächst eine fundamentale Einteilung des Begriffes Teil, nämlich die Einteilung in Stücke oder Teile im engsten Sinne, und in Momente oder abstrakte Teile des Ganzen. Jeden relativ zu einem Ganzen G selbständigen Teil nennen wir ein Stück, jeden relativ zu ihm unselbständigen Teil ein Moment (einen abstrakten Teil) dieses selben ganzen G. ${ }^{24}$

Husserl weist darauf hin, dass er den Begriff ,Teil“ „in dem weitesten Sinne“ verwendet, während das Wort ,Teil` im allgemeinen Sprachgebrauch auf dasjenige beschränkt ist, was Husserl als „relativ selbständigen Teil“ oder „Stück“ auffasst. Mereologisch gesehen, ist ,Teil‘ alles, was in einem - wiederum weit aufgefassten - ,Gegenstand‘ „unterscheidbar“ bzw. „,vorhanden“ ist (228). Momente sind Eigenschaften eines Gegenstandes; als Beispiel dafür kommt immer wieder die Farbe oder eine konkrete Färbung vor: „In der Einheit eines sinnlich Erscheinenden finden wir etwa die ganz bestimmte Rotfärbung als Moment und dann wieder das Gattungsmoment Farbe“ (227).

Der Unterschied zwischen Stück und Moment gründet auch darauf, dass ,,jedes phänomenale Ding und jedes Stück desselben“ im Unterschied zu einem Moment „getrennt

23 Als weitere Beispiele der sujetlosen Prosa nennt Šklovskij „die fast ganze Arbeit der Enzyklopädisten, den Essay unterschiedlichster Arten, die russische Publizistik“. Viktor Šklovskij: K technike vnesjužetnoj prozy, in: Literatura Fakta. Pervyj sbornik materialov rabotnikov LEFa, pod. red. N. F. Čužaka, Moskau 1929, 229-234, hier: 227, $232 \mathrm{f}$.

24 Husserl, Bd. 2 (Anm. 4), 266. Im folgenden Kapitel werden Zitate aus dieser Edition mit Seitenangaben direkt im Text nachgewiesen. 
vorstellbar“ sind (231). Husserls Beispiel für die Vorstellung eines Stücks ist die Zusammensetzung eines Pferdekörpers: „Wir können uns den Kopf eines Pferdes ,getrennt‘ oder ,für sich vorstellen, das heißt, wir können ihn in der Phantasie festhalten, während wir die übrigen Teile des Pferdes und die gesamte anschauliche Umgebung beliebig ändern und verschwinden lassen“ (ebd.). Die Unselbstständigkeit eines Moments wird erneut am Beispiel der Färbung verdeutlicht: „,[D]enn eine Färbung überhaupt und rein als solche kann nur als Moment in einem Gefärbten existieren“ (241). Ein Stück als „relativ selbständiger“ Teil kann dagegen auch „für sich, ohne Verknüpfung mit anderem“ existieren (253).

Wenn man den Unterschied zwischen Moment und Stück auf Prosa überträgt, tritt die Komplexität dieses Begriffes in seinen klassifikatorischen und kompositorischen Dimensionen besonders deutlich hervor. Eine Menge von Texten lässt sich klassifikatorisch als Prosa betrachten, wenn alle Texte mindestens ein gemeinsames Moment, das heißt mindestens eine gemeinsame Eigenschaft, besitzen. Der Begriff ,Prosa“ würde auch für dieses Moment stehen, wie „Spielarten der Prosa“ bei Lidija Ginzburg; ein minimales Moment des Prosaischen wäre die ,Ungebundenheit‘, die sich als solche nur in einem Text vorstellen lässt. Zugleich gibt es eine durch Walsers „Prosastücke“ und Čapeks „prózy“ explizierte Möglichkeit, eine Reihe von Texten als Stücke zu einer anderen Größe zusammenzufügen, die auch ,Prosa‘ wäre. Nun ist der Prosabegriff in ein Teil-GanzesVerhältnis auch im kompositorischen Sinne involviert und bezeichnet sowohl ein zusammengesetztes Ganzes als auch relativ selbstständige Teile desselben, die von ihm abgetrennt vorstellbar sind. Diese Stücke sind Teile eines Ganzen wie das Kompositionsgefüge eines Buches, zugleich können sie aber auch „für sich“ existieren und sogar vereinzelt in einer anderen Umgebung publiziert werden. Diese Idee von Prosastücken als Teile „einer langen handlungslosen Geschichte“ basiert aber wiederum auf einem abstrakten Moment der ,Prosa': auf einer Fortsetzungsdynamik des Prosaischen, die Grenzen zwischen Einzelteilen schaffen und sie als Schwellen durchdringen kann.

\section{„[P]rose book made up of sentences and paragraphs“}

Diese Fortsetzungsdynamik werde ich im nächsten Schritt auf einer dritten Ebene der Einteilungsproblematik von ,Prosa“ weiter verfolgen, nämlich in der sprachlichen Beschaffenheit eines Prosatextes. Im Zentrum der Aufmerksamkeit bleibt die Rolle des Prosabegriffes in literarischen Selbstreflexionen, nun am Beispiel von Gertrude Steins Essay Poetry and Grammar (1935), der mit einem kurzen Fragesatz ohne Fragezeichen beginnt: „What is poetry and if you know what poetry is what is prose“. ${ }^{25}$

25 Gertrude Stein: Poetry and Grammar, in: dies.: Lectures in America [1935], New York 1975, 209-246, hier: 209. Im folgenden Kapitel werden Zitate aus dieser Edition mit Seitenangaben direkt im Text nachgewiesen. 
Dieser poetologische Essay bietet epitextuelle Reflexionen zu mehreren Werken Steins, unter anderem zu ihrem Roman The Making of Americans: Being a History of a Family’s Progress, der zwischen 1903 und 1911 verfasst, aber erst 1925 vollständig veröffentlicht wurde. Die Entstehungsgeschichte dieses Romans gehört zur selben Epoche wie die Prosastücke von Robert Walser und der Brüder Čapek; ähnlich wie prózy von Richard Weiner und den Brüdern Čapek stand Steins Prosa im Kontext einer Rezeption des Kubismus. ${ }^{26}$ The Making of Americans rückt ein quantitatives Potenzial der Prosa zur Extension in den Mittelpunkt, denn der umfangreiche Roman umfasst circa tausend Seiten und wird in Poetry and Grammar als ,a long a very long prose book“ (228) bezeichnet.

Welche Dimension tritt hervor, wenn ein Roman als „Prosabuch“ betrachtet wird? Der Essaytitel von Stein deutet bereits eine „grammatische“ Perspektive an, zentral ist dabei die Syntax als textuelle Struktur. The Making of Americans ist auf den ersten Blick ein narrativer Familienroman, wie der Untertitel nahelegt: Being a History of $a$ Family's Progress. Das Prosabuch beinhaltet tatsächlich eine Geschichte der beiden Familien Hersland und Dehning in drei Generationen, allerdings wird die Narration durch Wiederholungen und Digressionen sowie durch innovative Syntax ständig herausgefordert. Wenn Stein von ihrer Schreiberfahrung an diesem Text erzählt, dominiert die Idee einer immerwährenden Fortsetzung als Schreibfluss: „When I first began writing, I felt that writing should go on, I still do feel that it should go on but when I first began writing I was completely possessed by the necessity that writing should go on“ (217). Auch die Konstruktion dieses Satzes führt die Idee einer textuellen Ausdehnung bzw. Bewegung performativ vor: Es werden immer weitere Teilsätze aneinandergereiht, die auf Wiederholungen basieren. Dadurch wird diejenige Bedeutung einer Bewegung nach vorne aktualisiert, die der Prosabegriff durch seine lateinische Etymologie in sich birgt: oratio pro(r)sa, das heißt ,vorwärtseilende Rede‘.

Steins Formulierung „writing should go on“ ist eine Hervorhebung der Schreibprozessualität, die sich dann in der Textbewegung wiederspiegelt. Der Essay Poetry and Grammar gehört zu einer Reihe von mehreren Epitexten, in denen sich Gertrude Stein mit dem Thema der Schreibpraxis auseinandersetzte: Dazu gehören auch How to Write (1931) und der Essay mit dem sprechenden Titel The Gradual Making of „The Making of Americans“ (1935). Wenn man die Vorwärtsausrichtung von pro(r)sa als „gradual making“ deutet, dann ist Prosa auch ein Praxisbegriff, der ein Bewusstsein für das Prosaschreiben (oder auch Prosasprechen in der rhetorischen Tradition) impliziert.

Diese Prozessualität ist bei Stein untrennbar von der Erschaffung einer Komposition aus Teilen. Der Roman The Making of Americans ist in keine Kapitel gegliedert;

26 Gertrude Stein war mit Pablo Picasso befreundet und hat mehrere Texte über ihn verfasst: zwei ,Wort-Portraits‘ Picasso (1909) und If I Told Him. A Completed Portrait of Picasso (1923) sowie den Essay Picasso (1938). 
seine fast tausend Seiten sind nach dem Inhaltsverzeichnis aus der Edition von 1925 nur in fünf Teile mit vier Zwischenüberschriften unterteilt. Im Essay Poetry and Grammar behauptet Stein, dass dieses Prosabuch aus Sätzen und Absätzen (,sentences and paragraphs“) besteht; hinzu kommt eine dritte Größe, die den Unterschied zwischen Satz und Absatz aufhebt. Das sind lange Sätze, die jeweils einen ganzen Absatz füllen - also eine syntaktische Periode: „When I wrote The Making of Americans I tried to break down this essential combination by making enormously long sentences that would be as long as the longest paragraph“ (223). Dementsprechend nennt Stein explizit drei Bausteine ihres „langen Prosabuches“: „The Making of Americans a long a very long prose book made up of sentences and paragraphs and the new thing that was something neither the sentence or the paragraph each one alone or in combination had ever done“ (228).

Solche Perioden sind auch Prosastücke bzw. Prosen; sie sind Teile und zugleich jeweils ein geschlossenes „ganzes Ding“: ,they had become something that was a whole thing and in so being they had a balance which was the balance of a space completely not filled but created by something moving“ (225). In diesem Zitat treffen sich Ideen von Ganzheit und Bewegung, Komposition und Prozessualität, die in Steins Prosakonzept einander ergänzen. Die Balance ist ein weiterer wichtiger Begriff, der Bewegung und Struktur in sich verbindet und zudem die syntaktische Beschaffenheit aus balancierten Sätzen und Perioden an eine epistemologische Dimension der Prosa anbindet. Es handelt sich nicht nur um Textualität, sondern auch um Erkenntnis, um eine Auseinandersetzung mit der Lebensbalance durch das Prosaschreiben: „I was writing The Making of Americans, I was completely obsessed by the inner life of everything including generations of everybody's living and I was writing prose, prose that had to do with the balancing the inner balancing of everything “ (235).

Das Prosakonzept ist bei Gertrude Stein auch mit einem mereologisches Modell von ,Prosa' als Stück und Moment zugleich verbunden: Ein umfangreicher Prosatext als Ganzes besteht aus kleineren Prosen, und das Zusammenfügen dieser Stücke zu einer Gesamtheit wird durch das Prosamoment als (syntaktische) Fortsetzungsmöglichkeit ermöglicht. Dieses verbindende Moment, unterstützt durch Wiederholung mehrerer Teilsätze, tritt dabei in Konkurrenz mit der einheitsstiftenden Funktion der narrativen Handlung, die in The Making of Americans zwar nicht abwesend, aber abgeschwächt ist. Eine große Rolle spielt in diesem Prosabuch wie bei Walser die Idee eines Neubeginns, die auf Störungen der narrativen Kohärenz hindeutet: „Alfred Hersland and Julia Dehning came to have some loving feeling and then they came to marrying. I am beginning again the history of them“. ${ }^{27}$ Stefana Sabin hat hervorgehoben, dass die „lose narrative Struktur in Gertrude Steins Prosa“ gerade „durch eine genau konstruierte

27 Gertrude Stein: The Making of Americans. Being a History of a Family’s Progress, Normal, IL 1999, 607. 
Sprache zusammengehalten“ wird. ${ }^{28}$ So entwickelt Prosa auch hier ihr Potenzial zur alternativen, ,sujetlosen` Textordnung, ohne auf Narration völlig zu verzichten.

\section{Prosa als mereologische Kategorie}

Diese Reihe von Beispielen aus unterschiedlichen Literaturen hat gezeigt, wie der Prosabegriff an der Schnittstelle von Poetologie und Schreibpraxis eine mereologische Problematik der Teil-Ganzes-Relationen mit sich bringt. Aus den induktiven Analysen ergeben sich die folgenden Thesen, die sich als Zusammenfassung und zugleich als Ausblick auf die weitere Erforschung der Prosa an einem umfangreicheren Material verstehen.

In den Prosabegriff selbst ist semantisch ein Verknüpfungsmodell eingeschrieben, das sich auch auf der textuellen Ebene erkennen lässt: Aus Prosen wird Prosa, wobei dieses Wort für einen Teil, für ein Ganzes und für eine gemeinsame Eigenschaft aller Teile zugleich steht. In Anlehnung an Edmund Husserls Lehre von den Ganzen und Teilen habe ich ,Prosa“ als Stück und Moment charakterisiert. Hinzu kommt eine dritte Dimension als Ganzheit besonderer Art: Im Prosabegriff ist ein Spannungsverhältnis zwischen Teilung und Bindung eines Ganzen impliziert. Diese Teilung in Prosastücke würde bei Husserl „Zerstückung“ heißen: „Stücke, die kein Stück identisch gemeinsam haben, nennen wir sich ausschließende (disjunkte) Stücke. Die Einteilung eines Ganzen in eine Mehrheit sich ausschließender Stücke nennen wir eine Zerstückung desselben““. ${ }^{29}$ Aus diesem Prozess der Zerstückung wird das Konzept eines „extensiven Ganzen“ abgeleitet:

Wenn ein Ganzes eine derartige Zerstückung zuläßt, daß die Stücke ihrem Wesen nach von derselben niedersten Gattung sind, als welche durch das ungeteilte Ganze bestimmt wird, so nennen wir es ein extensives Ganzes, seine Stücke extensive Teile. Hierher gehört beispielsweise die Teilung einer Ausdehnung in Ausdehnungen, spezieller einer Raumstrecke in Raumstrecken, einer Zeitstrecke in Zeitstrecken u. dgl. ${ }^{30}$

Dieser Definition entspricht auch die Teilung einer Prosa in Prosen, denn sowohl alle Stücke als auch das Ganze sind ,Prosa'. Die Ausdehnung und Fortbewegung des Prosatextes sowie die prozessuale Praxis des Prosaschreibens bzw. Sprechens können mit einer räumlichen oder zeitlichen Strecke verglichen werden. Das Wort ,Prosa' steht

28 Darin sieht Stefana Sabin auch eine Parallele zum Kubismus mit seiner „Akkumulation vieler Kompositionseinheiten“ und „Wiederholungen von geometrischen Elementen“. Stefana Sabin: ,... die genaue Ähnlichkeit ...“. Der ästhetische Sieg der Moderne und die Geschichte einer Freundschaft, in: Gertrude Stein: Picasso. Sämtliche Texte 1909-1938, Hamburg 2003, 113-135, hier: 125 f.

29 Husserl, Bd. 2 (Anm. 4), 267.

30 Ebd. 
also für ein „extensives Ganzes“ und für einen „extensiven Teil“ zugleich; in diesen Begriffen von Husserl wird das Kompositorische einer Teil-Ganzes-Struktur mit einer Dynamik der Extension verbunden. Dadurch tritt die Extensivität der Prosa hervor: ihr Potenzial, sich von einem kurzen Prosastück bis zu einem überlangen Prosabuch, beinahe bis in die Unendlichkeit zu erstrecken.

Diese mereologische Extensivität lässt sich auf drei Ebenen verfolgen. Eine basale Ebene des Prosaschreibens ist die Syntax mit solchen Teilen wie Satz, Absatz und Periode, die über ihre kompositionelle Bindungslogik und Ästhetik verfügen. Eine weitere Ebene umfasst unterschiedliche Formen der Zusammensetzung von (relativ) selbstständigen Texten in ein größeres Gefüge: von Montagetechniken bis zu unterschiedlichen Sammelformaten. Schließlich bietet der Prosabegriff ein heuristisches Instrument für die Konturierung variabler Gemeinschaften als Schnittmengen zwischen unterschiedlichen Diskursen und Wissensbereichen und zwischen Sachtext und Kunst. Solche Gemeinschaften basieren auf den jeweiligen verbindenden Momenten der Hybridität und sind „mögliche Ganze“ nach Husserl: „Jeder Gegenstand ist wirklicher oder möglicher Teil, $d$ h. es gibt wirkliche oder mögliche Ganze, die ihn einschließen“. ${ }^{31}$ Neben den textuellen Verbindungsmechanismen tritt hier die Performativität des Begriffes selbst hervor: seine integrative Kraft, eine gemeinsame textuelle Dimension unterschiedlicher Diskurse ins Zentrum der Aufmerksamkeit zu rücken.

Neben einer Einheitsstiftung durch fiktionale Handlung entwickelt die Prosakategorie das Potenzial, alternative syntaktische, kompositionelle und interdiskursive Ordnungsmodelle in der Literatur zu befördern. Prosa ist weniger ,ungebundene Rede، im wörtlichen Sinne, sondern , anders als Vers gebundene' Rede; zudem verfügt sie generell über eine Möglichkeit, zu einer ,anders gebundenen Rede` zu werden, sei es in Bezug auf Versdichtung, Narration oder tradierte Kompositionsmuster. ${ }^{32}$ Für die Erforschung solcher prosaischer Textordnungen und Kompositionsmechanismen bedarf es einer Mereologie der Prosa.

Edmund Husserls mereologische Lehre entstand im Rahmen des zweiten Bandes der Logischen Untersuchungen, der den Untertitel „Untersuchungen zur Phänomenologie und Theorie der Erkenntnis“ trägt. Wie Gertrude Steins Begriff der Balance als Charakteristik des Prosatextes und der wahrgenommenen Lebenswirklichkeit zugleich verdeutlichte, ist die Prosamereologie auch mit Erkenntnisprozessen und mit der Frage nach Wirklichkeitsstrukturen verbunden. Textuelle Teilungen und

\footnotetext{
31 Ebd., 226.

32 Diese Befreiung der Prosarede von der Versbindung bedeutete für die antike Rhetorik, dass „sich [Prosa] auch ohne Fesseln selbst das rechte Maß zu setzen weiß“ (Cicero). Klaus Weissenberger entwickelt dieses Argument zur These, dass das zweifache Wirkungsprinzip der Prosa im „Austragen der Spannung zwischen Anarchie, Chaos, Unbestimmtheit und Beliebigkeit einerseits und Kosmos, Norm, Notwendigkeit, Bestimmtheit, Gesetzmäßigkeit und Zwangsläufigkeit andererseits“ besteht. Weissenberger, Art. „Prosa“ (Anm. 2), 321 f., 325.
} 
Zusammensetzungen gehören zu einer „Suche nach der Realität“ (Lidija Ginzburg) im Sinne einer epistemologischen Verarbeitung der Wirklichkeit, zu deren „reinen oder formalen gegenständlichen Kategorien“ nach Husserl „Einheit, Vielheit, Anzahl, Beziehung, Verknüpfung usf.“ gehören. ${ }^{33}$

Dementsprechend kann Prosa als eine mereologische Kategorie definiert werden, die primär textuelle (An-)Ordnungsmodelle in den Vordergrund rückt und gerade dadurch zwischen , ungebundener Rede“ und menschlicher Erkenntnis vermittelt. Bereits in der Semantik des Prosabegriffes ist eine mereologische Dualität der Prosa in ihrer prozessualen Extension eingeschlossen: als ein produktives Spannungsverhältnis zwischen Teilung und Zusammensetzung, Integration und Pluralisierung, Singular und Plural.

33 Edmund Husserl: Logische Untersuchungen, Bd. 1, Prolegomena zur reinen Logik, 2., umgearb. Aufl., Halle (Saale) 1913, Nachdruck als 7. Aufl., Tübingen 1993, 244. 